\title{
Ophiopogonin D inhibiting epithelial NF-KB signaling pathway protects against experimental colitis in mice
}

\section{Liang Wang}

Dalian Medical University

Jiani Liu

Dalian Medical University

Liang Qiao

Dalian Medical University

Xiaoxiao Liao

Dalian Medical University

Huan Huang

Dalian Medical University

Jianyi Dong

Dalian Medical University

Jun Chen

Dalian Medical University

Dapeng Chen ( $\nabla$ cdp.9527@163.com )

Dalian Medical University https://orcid.org/0000-0001-9838-1646

Jingyu Wang

Dalian Medical University

\section{Research Article}

Keywords: Ophiopogonin D, colitis, inflammation, apoptosis, NF-kB

Posted Date: April 5th, 2021

DOl: https://doi.org/10.21203/rs.3.rs-261278/v1

License: (c) (1) This work is licensed under a Creative Commons Attribution 4.0 International License.

Read Full License 


\section{Abstract}

The sustained activation of the nuclear factor KB (NF-KB) signaling pathway has been observed in human inflammatory bowel disease (IBD). Ophiopogonin D (OP-D) is a small molecular compound isolated from Ophiopogon japonicus, a widely used herbal remedy. In this study, dextran sodium sulfate was used to make a mouse model of experimental colitis and verify the effect of OP-D on the mouse model of experimental colitis. Small molecule-protein molecular docking approaches were also used to discover the mechanisms underlying the OP-D-induced regulation of colitis.

In colitis, the OP-D can inhibit the apoptosis of intestinal mucosa cells, restore the intestinal barrier, and alleviate inflammation. The molecular docking simulations showed that OP-D had a high affinity with the REL-homology domain of NF-kB-p65 that affected its translocation to the nucleus. In a cell study, the effects of OP-D on inflammation and barrier dysfunction were significantly decreased by a small interfering RNA targeting NF-kB-p65. Further, the LPS-induced increase in NF-kB-p65 in the nucleus was also significantly inhibited by OP-D. OP-D alleviated experimental colitis by inhibiting NF-KB. New insights into the pathogenesis and treatment options of colitis are provided though this study.

\section{Introduction}

Inflammatory bowel disease (IBD) is a complex, multifactorial, recurrent gastrointestinal inflammatory disease, including ulcerative colitis (UC) and Crohn's disease (CD) [1].

In some western and newly industrialized countries, the incidence of IBD is on the rise [2,3]. Intestinal stricture and abscess often appear with patients who have IBD. Besides, severe patients may have some symptoms such as intestinal site bleeding and perforation, and even inflammation cancer transformation.[4-6]. Intestinal and extra-intestinal manifestations/complications cause mental and physical pain in patients with IBD during their active, young, and middle ages. Thus, IBD imposes heavy economic burdens on patients' families and society [7].

The pathology mechanism of colitis, which has not been fully revealed, has been considered to be a combination of genetic and environmental factors, as well as immune disturbances [8]. IBD often leads to changes in various cytokines (e.g., tumor necrosis factor (TNF- $a$ ), interleukin-6 (IL-6), and interleukin-1 (IL$1 \beta)$ ) in cells [9]. Nuclear factor KB (NF-KB) activation has been found to control the transcription of genes involved in immunity and inflammation in IBD [10]. The inhibition of the NF-KB-dependent signaling pathways represents a promising avenue for IBD therapy. The anti-inflammatory mechanisms of steroids for the treatment of IBD are dependent on the inhibition of NF-KB activation [11]. Steroids are also important for the treatment of moderate and severe IBD because common non-steroidal antiinflammatory drugs, including sulfasalazine, have limited effects on IBD [12, 13]. But steroids also carry risks, often with side effects, such as Cushing's syndrome. Steroids also suppress the production of adrenaline, leading to diabetes, glaucoma, osteoporosis, and even deadly infections [14]. Thus, it would be beneficial to discover a natural drug that can inhibit NF-KB activation for IBD treatment. 
As the main component of Ophiopogon japonicas, Ophiopogonin D (OP-D)has various biological functions, such as anti-tumor[15], enhancing immunity, and anti-oxidation[16]. OP-D can also inhibit cell apoptosis[17] and anti-inflammatory [18]. Among throat and intestinal diseases, OP-D can inhibit bacteria and inflammation and is included in a variety of traditional Chinese medicines that also treat inflammation-related diseases. The present study aimed to examine the effects of OP-D in an experimental mouse colitis model induced by the consumption of free drinking water containing dextran sodium sulphate (DSS). The potential therapeutic mechanisms were studied as well.

\section{Materials And Methods}

\section{Animal}

Ninety male C57BL/6J mice aged 6-8 weeks were provided by Dalian Medical University of China (license key: SYXK (Liao) 2018-0007). The mice weighed between 18 and 20g. The experimental protocol followed the guidelines of the Canadian Animal Protection Commission (CCAC) and the Guidelines for the Care and Use of Experimental Animals (National Academy of Sciences Press, 8th Edition). Animal research complies with ARRIVE guidelines [19].

\section{Reagents}

Obtained OP-D (purity: 98\%) from Shanghai Winherb Medical Technology Co.Ltd. (Shanghai, China). NFKB antibody, COX-2 antibody, inducible nitric oxide synthase (iNOS) antibody, Bcl-2 antibody, Bax antibody, caspase-3 antibody, myosin light chain kinase (MLCK) antibody and occludin antibody were purchased from Abcam (Hong Kong) Ltd. (Hong Kong, China). Other chemicals were obtained from Sigma-Aldrich (St. Louis, MO, USA)

\section{Cell Culture}

The cell line IEC-6 was obtained from the Cell Bank of Shanghai Research Institute (Shanghai, China). A high sugar DMEM medium containing $10 \%$ fetal bovine serum was used to culture the cells. An incubator was used to culture the IEC- 6 cells at $37^{\circ} \mathrm{C}$ with $5 \%$ carbon dioxide.

\section{Experimental Design}

40 C57BL/ 6 mice were randomly selected for the toxicity experiment of OP-D in vivo. To evaluate the effects of OP-D on colitis in vivo,50 C57BL/ 6 mice were randomly selected and divided into 5 groups of

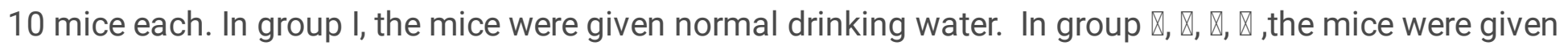

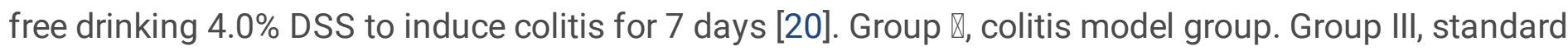
treatment control group-given Salicylazosulfopyridine (SASP); Group IV: OP-D low-dose group-given 10 mg/kg OP-D; Group V: OP-D high-dose group-given 40 mg/kg OP- D.

\section{Assessment of Inflammation}


We measured Bodyweight and disease index of each mouse daily[21]. Colitis disease activity index (DAI)a comprehensive score assessed by the percentage of weight loss, defecation, and stool bleeding according to previous studies[17].The colonic tissues of mice were collected after planned death. The colonic tissues were stained with hematoxylin and eosin. And immunohistochemical analysis was performed to evaluate the morphological structure of colonic tissues[22]. We measured the levels of TNFa, IL-1 $\beta$, and IL- 6 in the tissues by ELISA. The contents of malondialdehyde (MDA), glutathione (GSH), and superoxide dismutase (SOD)were determined by the method provided by the reagent supplier.

\section{Western Blot}

After obtaining the colon tissue of the mouse, it was quickly stored in liquid nitrogen. Total colonic tissue proteins were obtained with a protein extraction kit (KeyGen Biotech, China). SDS IPAGE technology was selected to isolate the proteins. We used methanol to activate the PVDF membrane for one minute. Next, the target protein was transferred to the PVDF membrane. The antibody was incubated at $4{ }^{\circ} \mathrm{C}$ overnight. A Multi-Spectral Imaging system (UVP, Cambridge, UK) was used to detect and quantify the bands.

\section{Cell Transfection}

According to the operating method provided by the reagent manufacturer, Lipofectamine 2000 (Invitrogen, Carlsbad, CA, USA) was used to transfect small molecule interfering RNA (siRNA) targeting NF-KB into IEC- 6 cells. Relevant experiments directly show the transfection efficiency, and based on it, IEC6 cells were cultured in a six-well cell plate, NF-KB siRNA and negative control were added to the cell culture medium, transfected for 48 hours to collect the cells. We extracted the total protein by the aforementioned method. The siRNA used in the experiment was obtained from Genepharma (Shanghai, China).

\section{Molecular Docking Simulation}

Molecular docking was conducted using MOE v2014.0901 software from Chemical Computing Group Inc. (Montreal, QC, Canada). The 2D structures of OP-D were drawn in ChemBioDraw 2014 and converted to the 3D structure in MOE by energy minimization. The 3D structure of the protein complex (NF-kB-p65) was obtained from the RCSB Protein Data Bank (PDB ID: 1IKN) [23]. Before docking, the force field of AMBER10: EHT and the implicit solvation model of the Reaction Field (R-field) were selected. MOE-Dock was used for molecular docking simulations of OP-D with NF-KB-p65. The docking workflow followed the "induced fit" protocol, in which the side chains of the receptor pocket were allowed to move according to ligand conformations, with a constraint on their positions. We used the weight that was 10 for tethering side-chain atoms to their original positions. For each ligand, all docked poses were ranked by London dG scoring first, then a force field refinement was carried out on the top 20 poses, followed by a rescoring of GBVI/WSA dG. The best (probable) binding modes have the lowest free energies of binding. Molecular graphics were generated by PyMOL software.

\section{Statistical Analysis}


The experimental data were analyzed by single-blind. One-way analysis (ANOVA) was used for comparison between groups, and Student' t-test was used to verify the gap between the data in the groups. All displayed values are calculated with the mean and standard deviation (SD). Kaplan-Meler was used for further evaluation and rank-sum tests. All experimental values of the above analysis are obtained by repeating the experiment more than 6 times. $P<0.05$ was considered to be statistically significant.

\section{Results}

\section{Dose/Concentration Selection and Toxicity of OP-D in Normal Cells and Mice}

Fig. 1a shows the chemical structure of OP-D. Based on the cytotoxicity test in vitro, the effective and non-toxic concentration of OP-D in subsequent experiments were determined. Exposure to $20 \mu \mathrm{mol} / \mathrm{L} \mathrm{OP-}$ $\mathrm{D}$ for $24 \mathrm{~h}$ had no remarkable effect on cell viability (Fig. 1b). Intragastric administration of OP-D (10 $\mathrm{mg} / \mathrm{kg}$ and $40 \mathrm{mg} / \mathrm{kg}$ ) for seven consecutive days had no significant effects on mouse body weight (Fig.1c), the weight ratio of the spleen to the body (Fig.1d), or cytokine profiles (IL-6, IL-1 3 , and TNF-a) (Fig. 1e-g) of colon tissue. Besides, the expression of NF-kB-p65 was not significantly affected by the intragastric administration of OP-D (Fig. 1h). Because of the experiments and results previously provided by other researchers $[24,25], \leq 40 \mathrm{mg} / \mathrm{kg}$ and $\leq 20 \mu \mathrm{mol} / \mathrm{L}$ OP-D were used in vivo and in vitro experiments, respectively.

\section{OP-D Alleviated Colitis Symptoms}

The colitis model induced by DSS was successfully established, which was certified by biochemical and macro-analysis of significant colonic mucosal lesions, including severe ulceration, congestion, and edema. On the surface of the colonic mucosa, many ulcers covered with grimy sphacelus. HE staining showed a variety of obvious symptoms of inflammation, including markedly thickened muscular layer, neutrophil infiltration, disordered gland arrangement, and crypt abscess (Fig.2a). The gross morphology of the colon (Fig.2b) indicated that OP-D alleviated the inflammatory response. All the colitis symptoms induced by $4.0 \%$ DSS, including shorter colon length, decreased bodyweight, as well as disease activity index was reversed by OP-D. (Fig.2c-e)

\section{OP-D Attenuated Oxidative Stress in the Colitis}

The effects of OP-D on oxidative stress were studied in the colitis model. Compared to the sham group, colonic MDA was increased, while GSH and SOD were decreased in the colitis mice. However, such variations in colonic mice were reversed by OP-D treatment (Fig.3a-C). Those results suggested that OP-D had protective effects against oxidative damage in the colitis model.

\section{OP-D Ameliorated Responses of Inflammation in Colitis}


The levels of TNF- $a$, IL-1 $\beta$, and IL- 6 were elevated in the colitis model. After treatment of seven days, OP-D remarkably relieved symptoms of colitis(Fig.3d-f). The iNOS and COX-2 were increased in the colitis model, which was also remarkably reversed by OP-D (Fig.4a, d, and e).

\section{OP-D Decreased Epithelial Apoptosis and Restored Intestinal Epithelial Barrier Dysfunction During Colitis}

OP-D treatment for seven days reversed changes, including Bax and cleaved caspase3 (cl-caspase3) that were the apoptosis-related proteins, in colitis mice, was significantly increased, while Bcl-2 was remarkably decreased (Fig.4a, c, f, and h). Occludin was significantly decreased in the colitis model, while the MLCK that was the protein of epithelial cells related to tight junction dysfunction, was remarkably increased. After seven days of treatment, those changes were also reversed by OP-D (Fig.4a, b, and g).

\section{Potential Mechanisms Underlying the OP-D-Induced Therapeutic Effects in the Colitis Model}

The expression of NF-KB-p65 was significantly increased in colitis compared to the sham group indicated in immunohistochemical staining, which was reversed by OP-D treatment (Fig.5a). However, OP-D treatment for seven days reversed those increases remarkably (Fig. $5 b)$. In IEC- 6 cells, LPS $(2 \mu \mathrm{g} / \mathrm{mL})$ induced a significant increase in cl-caspase3, COX-2, and MLCK, which reflected the induction of inflammation and epithelial barrier dysfunction. OP-D $(20 \mu \mathrm{mol} / \mathrm{L})$ reversed the LPS-induced increase in cl-caspase3, iNOS, and MLCK, which was remarkably blocked by a siRNA targeting NF-kB-p65 (Fig.5c-g). Those results suggested that the inhibition of the NF-KB-p65 signaling pathway may be involved in the OP-D induced treatment of DSS colitis.

\section{Molecular docking analysis of the binding mode of OP-D with NF-KB-p65}

To investigate how OP-D affected the function of NF-KB-p65, the docking mode of OP-D with NF-KB-p65 was established. The docking score of OP-D for NF-KB-p65 was $-8.99 \mathrm{kcal} / \mathrm{mol}$, which indicated that OP-D had a high affinity for NF-kB-p65. The mouse NF-kB-p65 consisted of 549 amino acids. The binding site of NF-KB-p65 with OP-D was the N-terminal REL-homology domain (RHD) including amino acids 19-306. The RHD is involved in DNA binding and inhibitor interactions, which also assists with the translocation of NF-KB into the nucleus [26]. Through docking simulation studies, we found that OP-D interacted with Ser51, Arg273, Glu225, and Phe239 of NF-kB-p65 via hydrogen bond interactions (Fig. 6a-c). The computational result indicated that OP-D bound to the RHD domain of NF-KB-p65 and may block NF-kBp65 translocation to the nucleus. The translocation of NF-KB-p65 to the nucleus was assessed to confirm the computational results by immunofluorescence. In IEC- 6 cells incubated with $2 \mu \mathrm{g} / \mathrm{mL}$ LPS, 5 and 20 $\mu \mathrm{mol} / \mathrm{L}$ OP-D significantly inhibited the nuclear translocation of NF-KB-p65 that was increased in the model (Fig. 6d). The docking results were consistent with those results and confirmed that OP-D directly bound to the RHD domain of NF-kB-p65 to block its translocation to the nucleus. NF-kB signaling was inhibited by such inhibitory activities.

\section{Discussion}


In this study, the effects of OP-D on colitis and the potential mechanisms were studied. We demonstrated for the first time that OP-D reversed the pathological changes and alleviated colitis symptoms, mucosal barrier dysfunction, inflammatory responses, epithelial cell apoptosis, and oxidative stress in a mouse colitis model through inhibiting the NF-KB signaling pathway. OP-D bound to the N-terminal RHD domain of NF-KB-p65 to inhibit its activity.

According to previous research, the abnormal expression of inflammatory cytokines, such as IL-6, TNF-a, and IL-1 $\beta$, is an important cause of colitis, and that such inflammatory cytokines are increasingly expressed in IBD patients and play important roles in the pathogenesis of IBD $[27,28]$. In colitis, activated intestinal inflammatory cells produce cytokines and metabolize excess reactive oxygen species (ROS). Excess ROS can destroy oxidizable biomolecules reversibly or irreversibly, which lead to apoptosis and inflammation. Increased intestinal epithelial cell apoptosis has been detected at acute inflammatory sites in subjects with UC [29]. Excessive apoptosis of epithelial cells can damage the integrity of the intestinal barrier, which is essential for protecting against luminal pathogens.

In this study, OP-D significantly inhibited inflammatory responses in a DSS-induced colitis model, with the decrease of cytokine expressions, including IL-1 $\beta$, TNF- $a$, and IL-6, which are in agreement with previous study[30,31] . OP-D inhibited Serum MDA and GSH activities in ovariectomized osteoporosis model mice[32]. likewise, in our study, the excessive oxidative stress in DSS-induced colitis was also suppressed by OP-D, which was confirmed by decreases in the levels of MDA and GSH and increases in the levels of SOD. Symptoms of the damaged intestinal barrier function, including excessive apoptosis and the reduction of tight junction proteins, were also reversed by OP-D. All such results suggested that OP-D exerted therapeutic effects on DSS-induced colitis, which was confirmed by the reduction of the response of inflammation and oxidative stress, and the recovery of the barrier function of intestines.

OP-D exerted multiple pharmacological effects and many potential targets were involved. This study aimed to detail the mechanisms of the OP-D-induced treatment effects on colitis. Studies have shown that the NF-KB pathway is related to all factors, including inflammation, the oxidative stress, apoptosis, and the recovery of the barrier function of intestines [33]. NF-KB activation is required for apoptosis in fibrocystin/polyductin-depleted kidney epithelial cells, and CFTR ameliorates high glucose-induced oxidative stress and inflammation by mediating the NF-KB and MAPK signaling pathways in endothelial cells as well as arsenic downregulates tight junction claudin proteins through p38 and NF-kB in the intestinal epithelial cell line, HT-29 [34, 10,35]. Based on previous studies, OP-D exerts a positive effect on accommodating cardiovascular hypertophy through inhibiting the expression of NF-KB by regulating CYP2J3 to suppress inflammation[36]. These factors are consistent with the reversal of intestinal inflammation by OP-D, which guides the study of the potential mechanism of OP-D treatment of colitis. In the cell study, OP-D decreased cell apoptosis, while the recovery of the intestinal barrier function was significantly inhibited by a siRNA targeting NF-KB-p65. We found that NF-KB-p65 might be a potential target of OP-D for the treatment of colitis. The NF-KB signaling pathway has been well studied in the pathogenesis of IBD and is often imbalanced in patients, leading to the abnormal production of cytokines in the intestine [37]. 
The docking results suggested that OP-D had a high affinity for the RHD domain of NF-kB. That domain was involved in DNA binding, protein dimerization, and IKB binding. The RHD also contained a nuclear localization signal (NLS) sequence, which was necessary for the activation of NF-KB in the nucleus. In the cytoplasm, NF-KB binds to IKB protein. IKB masks the NLS of NF-KB and retains it in the cytoplasm. When IKB is degraded, the NLS of NF-KB is exposed, allowing it to enter the nucleus and bind target genes. The experimental results further confirmed that OP-D significantly inhibited LPS-induced translocation of NFKB. Several previous studies by other scholars also suggest that OP-D blocked the nuclear translocation of NF-KB-p65 to suppress inflammation in vivo[36]. All such results suggested that the addition of OP-D significantly inhibited the translocation of NF-KB induced by LPS.

\section{Conclusion}

Collectively, we found that OP-D significantly alleviated colitis symptoms by directly binding and inhibiting NF-KB-p65 enter the nucleus. The exact details of the OP-D interaction with the RHD of NF-KBp65, and whether other mechanisms are also involved in OP-D reductions in colitis, require further study. In IBD, the novel insights into the mechanisms and potential therapeutic use of OP-D could be provided by the results of the present study.

\section{Abbreviations}

Bcl-2, B-cell lymphoma-2; CD, Crohn's disease; COX-2, Cyclooxygenase-2; DSS, dextran sodium sulphate; ELISA, Enzyme-linked immunosorbent assay; IBD, inflammatory bowel disease; IL-6,Interleukin 6;LPS, Lipopolysaccharide;MDA, malondialdehyde; MLCK, myosin light chain kinase; NF-KB, Nuclear factor KB; OP-D, Ophiopogonin D; SASP, salicylazosulfapyridine; TNF, Tumor necrosis factor; UC, ulcerative colitis;

\section{Declarations}

\section{FUNDING INFORMATION}

This work was supported by the National Key Research and Development Project of China (2017YFD050160203), National Natural Science Foundation of China (81600440).

\section{COMPLIANCE WITH ETHICAL STANDARDS}

\section{Compliance and ethics}

All studies involving animals conducted in accordance with the Animal Protection Council of Canada (CCAC) guidelines or guidelines for the Care and Use of Laboratory Animals (8th edition, National Academy of Sciences press).

\section{Conflicts of Interest}

The authors declare no conflict of interest. 
Availability of data and material

The data used to support the findings of this study are available from the corresponding author upon request.

\section{Code availability}

the materials might remain accessible for 10 years, or "permanently".

\section{Authors' contributions}

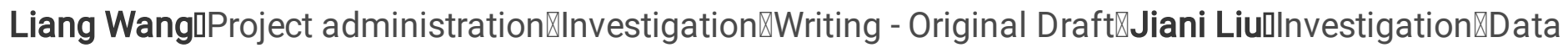

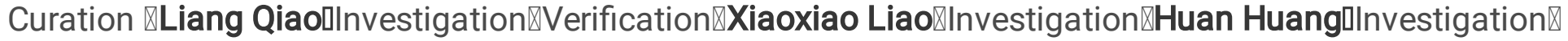

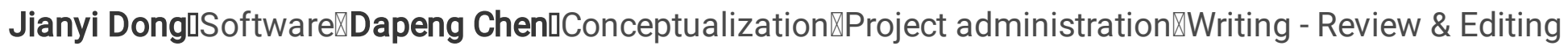
$\square$ Jingyu Wang $\square$ Resources $₫$ Supervision $\otimes$ Funding acquisition.

\section{Ethics approval}

This study was approved by the animal experiment ethics committee of Dalian Medical University.There is no human ethical problem in this study.

\section{Consent to participate}

All authors agreed with the content, all gave explicit consent to submit and that we obtained consent from the responsible authorities at the institute.

\section{Consent for publication}

All participants signed the published informed consent.

\section{References}

1. Ponder, A., and M. D. Long. 2013. A clinical review of recent findings in the epidemiology of inflammatory bowel disease. Clin Epidemio/ 5:237-247. doi:10.2147/CLEP.S33961.

2. Figueredo, C. M., A. P. Martins, R. Lira-Junior, J. B. Menegat, A. T. Carvalho, R. G. Fischer, and A. Gustafsson. 2017. Activity of inflammatory bowel disease influences the expression of cytokines in gingival tissue. Cytokine 95:1-6. doi:10.1016/j.cyto.2017.01.016.

3. Guan, Q., and J. Zhang. 2017. Recent Advances: The Imbalance of Cytokines in the Pathogenesis of Inflammatory Bowel Disease. Mediators Inflamm 2017:4810258. doi:10.1155/2017/4810258.

4. b, Fabio Vieira Teixeira a, Rogerio Saad Hosne c, and Carlos Walter Sobrado d \%J Journal of Coloproctology. 2015. Management of ulcerative colitis: a clinical update. 35 (4):230-237.

5. Neurath, M. 2017. Current and emerging therapeutic targets for IBD. Nat Rev Gastroenterol Hepatol 14 (11):688. doi:10.1038/nrgastro.2017.138. 
6. Sun, J., and I. Kato. 2016. Gut microbiota, inflammation and colorectal cancer. Genes Dis 3 (2):130143. doi:10.1016/j.gendis.2016.03.004.

7. Kaplan, G. G. 2015. The global burden of IBD: from 2015 to 2025 . Nat Rev Gastroenterol Hepatol 12 (12):720-727. doi:10.1038/nrgastro.2015.150.

8. Guan, Q. 2019. A Comprehensive Review and Update on the Pathogenesis of Inflammatory Bowel Disease. J Immunol Res 2019:7247238. doi:10.1155/2019/7247238.

9. Abraham, C., and J. H. Cho. 2009. Inflammatory bowel disease. N Engl J Med 361 (21):2066-2078. doi:10.1056/NEJMra0804647.

10. Fei, Y., L. Sun, C. Yuan, M. Jiang, Q. Lou, and Y. Xu. 2018. CFTR ameliorates high glucose-induced oxidative stress and inflammation by mediating the NF-kappaB and MAPK signaling pathways in endothelial cells. Int J Mol Med 41 (6):3501-3508. doi:10.3892/ijmm.2018.3547.

11. Probert, C. 2013. Steroids and 5-aminosalicylic acids in moderate ulcerative colitis: addressing the dilemma. Therap Adv Gastroenterol 6 (1):33-38. doi:10.1177/1756283X12461395.

12. Chen, P., D. Bakke, L. Kolodziej, J. Lodolce, C. R. Weber, D. L. Boone, and F. G. Toback. 2015. Antrum Mucosal Protein-18 Peptide Targets Tight Junctions to Protect and Heal Barrier Structure and Function in Models of Inflammatory Bowel Disease. Inflamm Bowel Dis 21 (10):2393-2402. doi:10.1097/MIB.0000000000000499.

13. Sykes, Lynne, Kacie R Thomson, Emily J Boyce, S Lee Yun, and Phillip R \%J Immunology Bennett. 2015. Sulfasalazine augments a pro-inflammatory response in IL-1 $\beta$-stimulated amniocytes and myocytes. 146 (4).

14. Pharmacology, P. J. Rutgeerts \%J Alimentary, and Therapeutics. 2001. The limitations of corticosteroid therapy in Crohn's disease.

15. Olofsson, K. M., B. Hjertner, C. Fossum, C. M. Press, and R. \%J Veterinary Journal Lindberg. 2015. Expression of T helper type 17 (Th17)-associated cytokines and toll-like receptor 4 and their correlation with Foxp3 positive cells in rectal biopsies of horses with clinical signs of inflammatory bowel disease.

16. Ma, X. Y., X. X. Wen, X. J. Yang, D. P. Zhou, Q. Wu, Y. F. Feng, H. J. Ding et al. . 2018. Ophiopogonin D improves osteointegration of titanium alloy implants under diabetic conditions by inhibition of ROS overproduction via Wnt/beta-catenin signaling pathway. Biochimie 152:31-42. doi:10.1016/j.biochi.2018.04.022.

17. Yan, Zhiyu, Guang Liu, Min Liang, and Yanjun Xu. 2018. Ophiopogonin D inhibits cell proliferation and induces apoptosis of human laryngocarcinoma through downregulation of cyclin $\curvearrowright \mathrm{B} 1$ and MMP-9 and upregulation of p38-MAPK signaling. Oncology Letters. doi:10.3892/ol.2018.9788.

18. Huang, Xiaoyan, Yuguang Wang, Zhaoyan Zhang, Yuan Wang, Xiangmei Chen, Yi Wang, and Yue \%J Biochem Biophys Res Commun Gao. 2017. Ophiopogonin D and EETs ameliorate Ang II-induced inflammatory responses via activating PPARa in HUVECs.123.

19. Kilkenny, C., W. Browne, I. C. Cuthill, M. Emerson, D. G. Altman, Refinement National Centre for the Replacement, and Research Reduction of Amimals in. 2011. Animal research: reporting in vivo 
experiments--the ARRIVE guidelines. J Cereb Blood Flow Metab 31 (4):991-993. doi:10.1038/jcbfm.2010.220.

20. Xiong, Y., J. Wang, H. Chu, D. Chen, and H. Guo. 2016. Salvianolic Acid B Restored Impaired Barrier Function via Downregulation of MLCK by microRNA-1 in Rat Colitis Model. Front Pharmacol 7:134. doi:10.3389/fphar.2016.00134.

21. Schwanke, Raquel Cristina, Rodrigo Marcon, Flavia Carla Meotti, Allisson Freire Bento, Rafael Cypriano Dutra, Moacir Geraldo Pizzollatti, and João B. Calixto. 2013. Oral administration of the flavonoid myricitrin prevents dextran sulfate sodium-induced experimental colitis in mice through modulation of PI3K/Akt signaling pathway. Molecular Nutrition \& Food Research 57 (11):1938-1949. doi:10.1002/mnfr.201300134.

22. Ghia, J. E., P. Blennerhassett, and S. M. Collins. 2008. Impaired parasympathetic function increases susceptibility to inflammatory bowel disease in a mouse model of depression. J Clin Invest 118 (6):2209-2218. doi:10.1172/JCl32849.

23. Huxford, T. , ., D B Huang, S. Malek, ., and G. Ghosh, . 1998. The crystal structure of the IkappaBalpha/NF-kappaB complex reveals mechanisms of NF-kappaB inactivation. Cel/95 (6):759770 .

24. Zhang, Y. Y., C. Meng, X. M. Zhang, C. H. Yuan, M. D. Wen, Z. Chen, D. C. Dong, Y. H. Gao, C. Liu, and Z. Zhang. 2014. Ophiopogonin D Attenuates Doxorubicin-Induced Autophagic Cell Death by Relieving Mitochondrial Damage In Vitro and In Vivo. Journal of Pharmacology and Experimental Therapeutics 352 (1):166-174. doi:10.1124/jpet.114.219261.

25. Zhang, Yuanyuan, Yuwei Han, Kefeng Zhai, Minhui Sun, Jihua Liu, Boyang Yu, and Junping Kou. 2015. Ophiopogonin-D suppresses MDA-MB-435 cell adhesion and invasion by inhibiting matrix metalloproteinase-9. Molecular Medicine Reports 12 (1):1493-1498. doi:10.3892/mmr.2015.3541.

26. Lin-Feng, Chen, and Warner C Greene. 2004. Shaping the nuclear action of NF-kappaB. Nature Reviews Molecular Cell Biology 5 (5):392-401.

27. Sipos, F., T. Zagoni, B. Molnar, and Z. Tulassay. 2002. [Changes in the proliferation and apoptosis of colonic epithelial cells in correlation with histologic activity of ulcerative colitis]. Orv Hetil 143 (44):2485-2488.

28. Shkoda, A., T. Werner, H. Daniel, M. Gunckel, G. Rogler, and D. Haller. 2007. Differential protein expression profile in the intestinal epithelium from patients with inflammatory bowel disease. $J$ Proteome Res 6 (3):1114-1125. doi:10.1021/pr060433m.

29. Neurath, M. F. 2014. Cytokines in inflammatory bowel disease. Nat Rev Immunol 14 (5):329-342. doi:10.1038/nri3661.

30. Qian, J., F. Jiang, B. Wang, Y. Yu, X. Zhang, Z. Yin, and C. Liu. 2010. Ophiopogonin D prevents H2O2induced injury in primary human umbilical vein endothelial cells. J Ethnopharmacol 128 (2):438-445. doi:10.1016/j.jep.2010.01.031.

31. Huang, X., Y. Wang, Z. Zhang, Y. Wang, X. Chen, Y. Wang, and Y. Gao. 2017. Ophiopogonin D and EETs ameliorate Ang II-induced inflammatory responses via activating PPARalpha in HUVECs. 
Biochem Biophys Res Commun 490 (2):123-133. doi:10.1016/j.bbrc.2017.06.007.

32. Huang, Q., B. Gao, L. Wang, H. Y. Zhang, X. J. Li, J. Shi, Z. Wang et al. . 2015. Ophiopogonin D: A new herbal agent against osteoporosis. Bone 74:18-28. doi:10.1016/j.bone.2015.01.002.

33. Kolesov, S. A., L. V. Korkotashvili, A. B. Yazykova, E. N. Fedulova, O. A. Tutina, and N. I. Tolkacheva. 2013. S-nitrosothiols, nitric oxide and proinflammatory cytokines in children with inflammatory bowel disease. Clin Lab 59 (9-10):953-957. doi:10.7754/clin.lab.2012.120632.

34. Mangolini, A., M. Bogo, C. Durante, M. Borgatti, R. Gambari, P. C. Harris, R. Rizzuto, P. Pinton, G. Aguiari, and L. del Senno. 2010. NF-kappaB activation is required for apoptosis in fibrocystin/polyductin-depleted kidney epithelial cells. Apoptosis 15 (1):94-104. doi:10.1007/s10495009-0426-7.

35. Jeong, C. H., J. S. Seok, M. C. Petriello, and S. G. Han. 2017. Arsenic downregulates tight junction claudin proteins through p38 and NF-kappaB in intestinal epithelial cell line, HT-29. Toxicology 379:31-39. doi:10.1016/j.tox.2017.01.011.

36. Wang, Y., X. Huang, Z. Ma, Y. Wang, X. Chen, and Y. Gao. 2018. Ophiopogonin D alleviates cardiac hypertrophy in rat by upregulating CYP2J3 in vitro and suppressing inflammation in vivo. Biochem Biophys Res Commun 503 (2):1011-1019. doi:10.1016/j.bbrc.2018.06.110.

37. Atreya, I., R. Atreya, and M. F. Neurath. 2008. NF-kB in inflammatory bowel disease. Journal of Internal Medicine 263 (6):591-596. doi:10.1111/j.1365-2796.2008.01953.x.

\section{Figures}



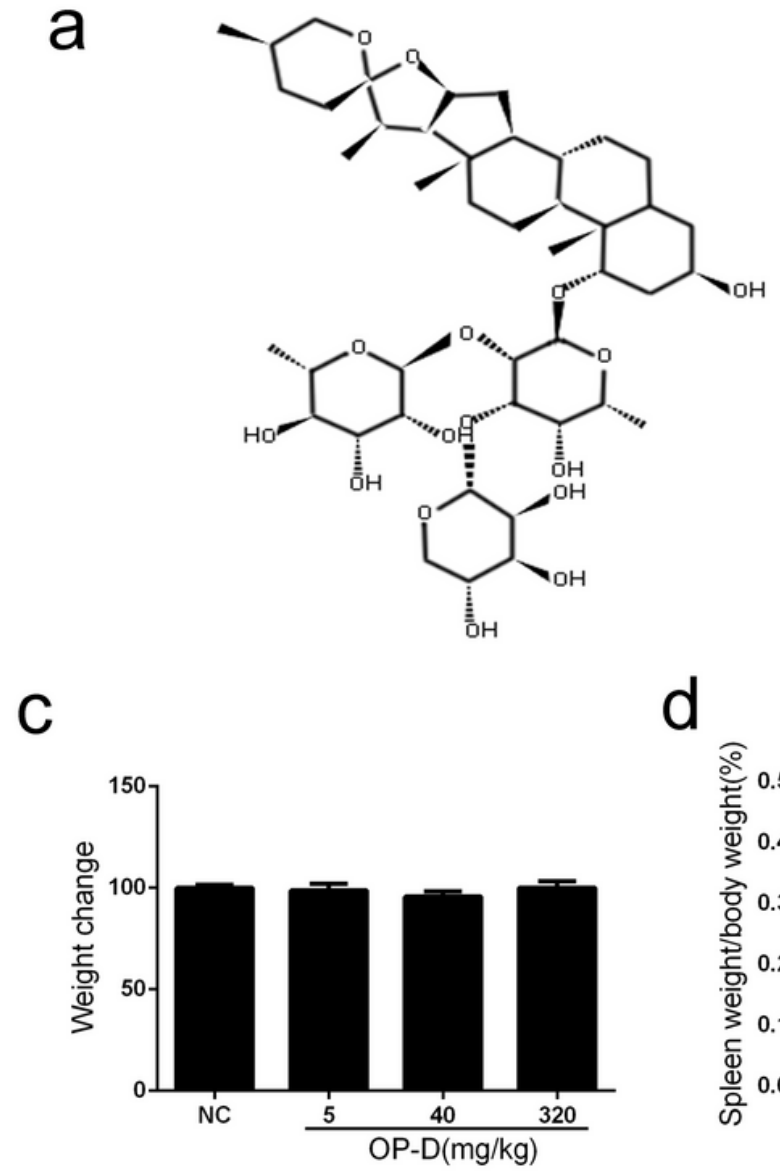

d

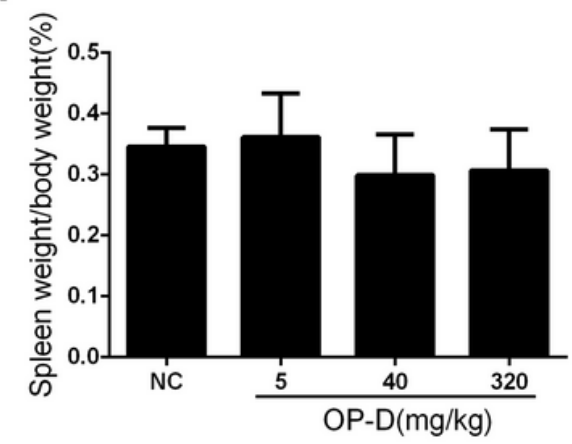

9

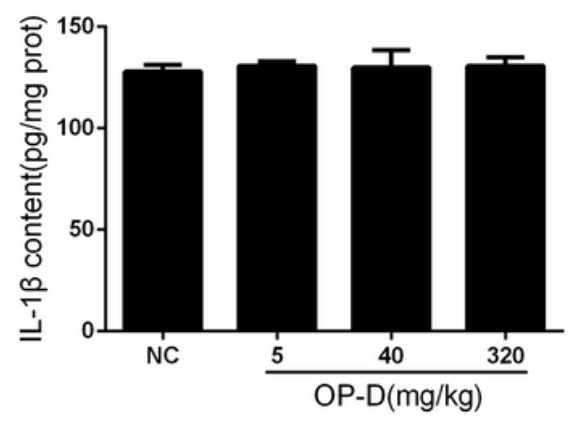

b

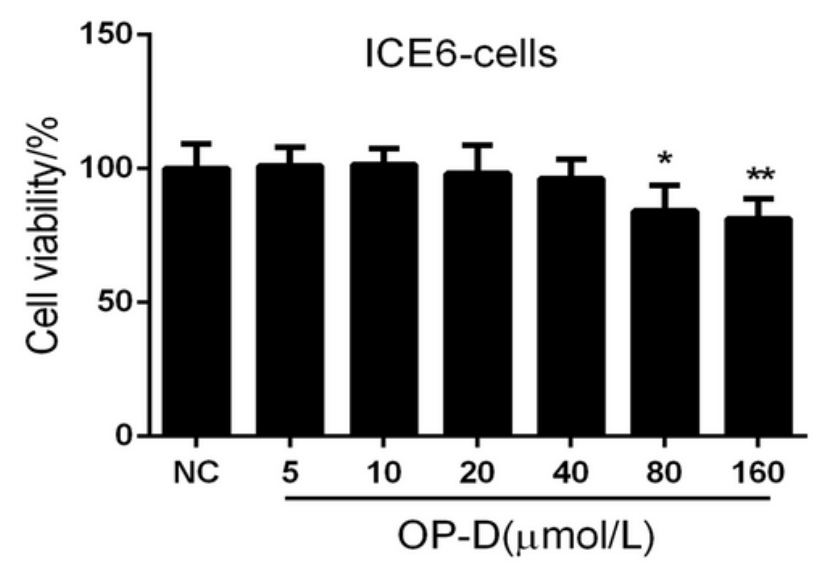

e

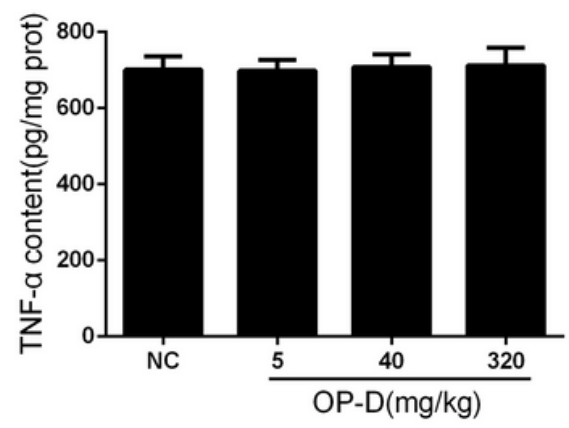

(kDa)

$\mathrm{h}$
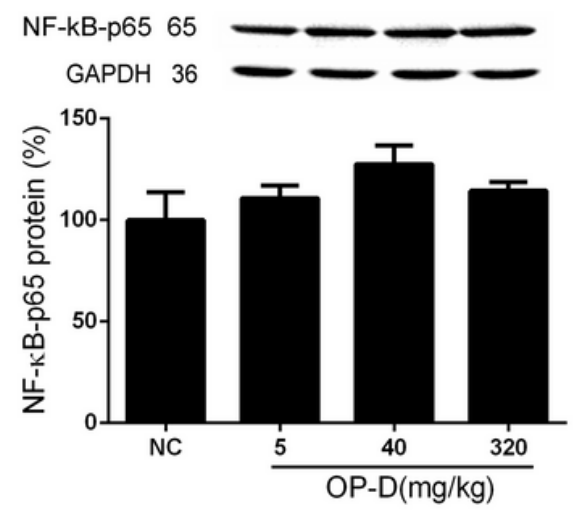

Figure 1

Dose/concentration selection of OP-D in normal cells and mice. (a) chemical structure of OP-D. (b) The effect of OP-D on IEC- 6 cells was studied using the CCK8 assay. OP-D was administered once a day for seven consecutive days by gavage. Mice body weights (c) and the ratio of spleen weight to body weight(d)were recorded. The expression levels of colonic cytokines, TNF- $a(e), I L-1 \beta(f)$, and IL- 6 (g) were examined by ELISA. The expression of NF-KB-p65 (h) was also studied by western blotting. Data were expressed as the mean \pm SD. The data in the sham group is set to $100 \%$, and other data is set to the relative value of sham. $\mathrm{N}=6$ in $\mathrm{B}-\mathrm{H},{ }^{\star} \mathrm{p}<0.05,{ }^{* \star} \mathrm{p}<0.01$, compared to the sham group. 

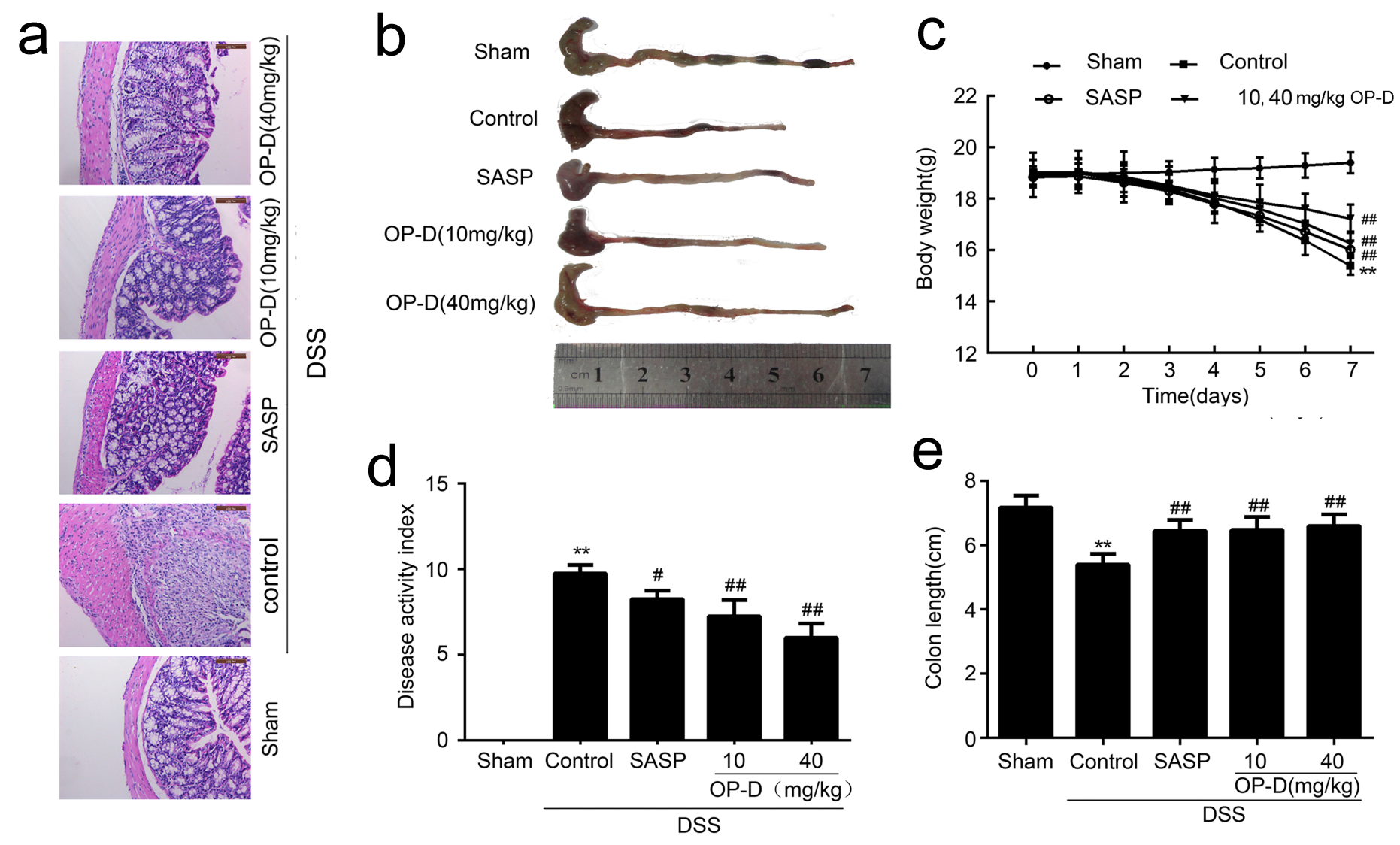

Figure 2

OP-D alleviated colitis symptoms. Colitis symptoms were examined after seven days of OP-D $(10 \mathrm{mg} / \mathrm{kg}$ and $40 \mathrm{mg} / \mathrm{kg}$ ) treatment. (a) HE-staining of mouse colonic tissue (100 on). Gross morphology of colon tissue (b). Effects of OP-D on (c) body weight, (d) disease activity index, and (e) colon length in colitis mice. Data were expressed as the mean \pm SD. The data in the sham group is set to $100 \%$, and other data is set to the relative value of sham. ${ }^{*} P<0.05,{ }^{*} P<0.01$, compared to the sham group $(n=6$ mice); $\# P<0.05$, $\# \# P<0.01$, compared to the DSS-colitis control group ( $\mathrm{n}=6$ mice) 

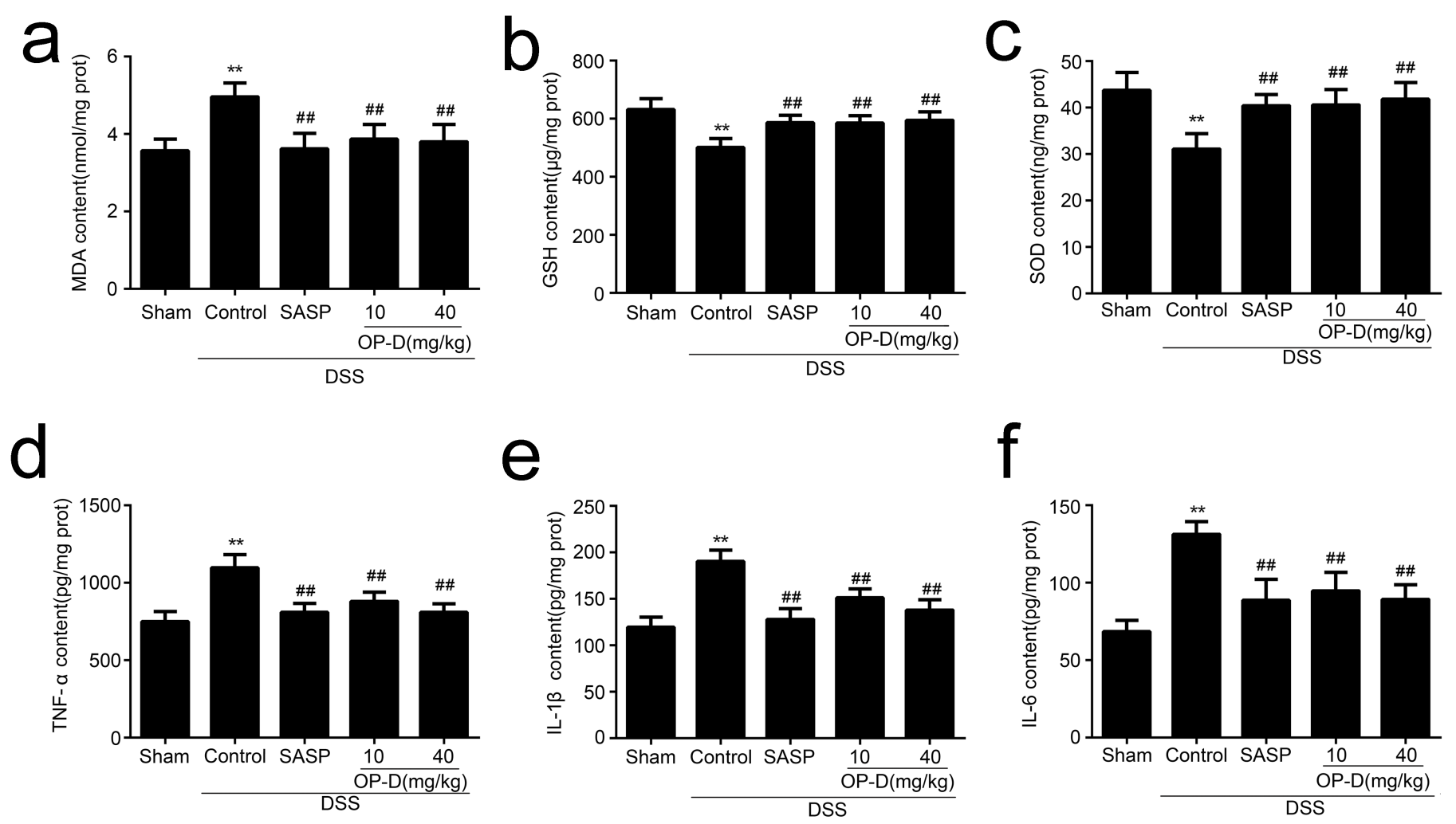

\section{Figure 3}

OP-D attenuated oxidative stress, neutrophil infiltration, and cytokine profiles. Effects of OP-D $(10 \mathrm{mg} / \mathrm{kg}$ and $40 \mathrm{mg} / \mathrm{kg}$ ) on (a) MDA, (b) GSH, (c) SOD, (d)TNF-a, (e) IL-1 3 , and (f) IL-6 in mice colon tissue, as determined by ELISA. Data are expressed as the mean \pm SD. The data in the sham group is set to $100 \%$, and other data is set to the relative value of sham. ${ }^{*} P<0.05,{ }^{*} \mathrm{P}<0.01$, compared to the sham group ( $\mathrm{n}=6$ mice); $\# \mathrm{P}<0.05, \# \# \mathrm{P}<0.01$, compared to the DSS-colitis control group ( $\mathrm{n}=6$ mice). 
a
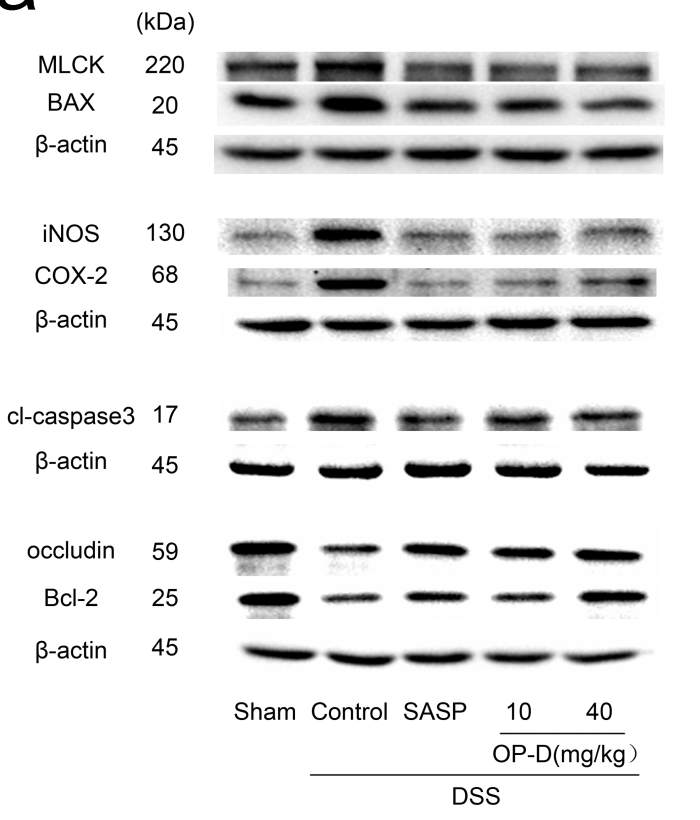

f

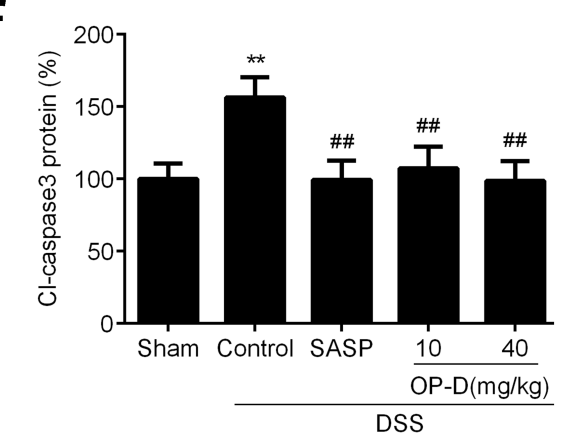

b

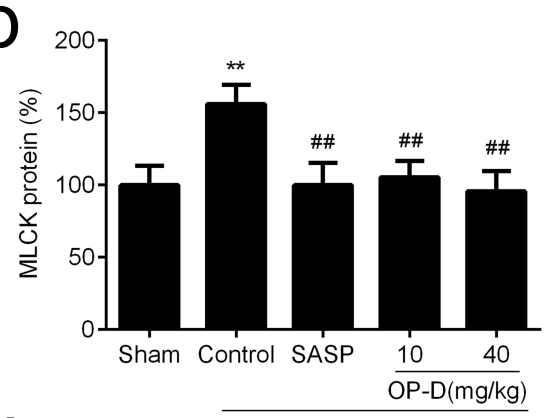

d

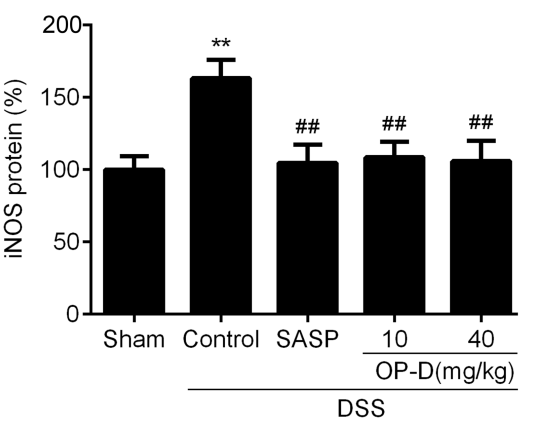

9

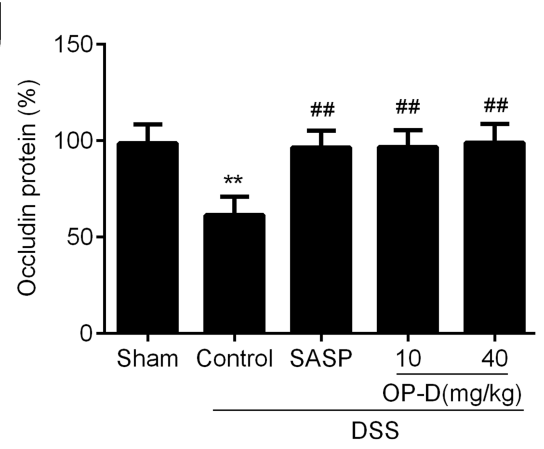

C
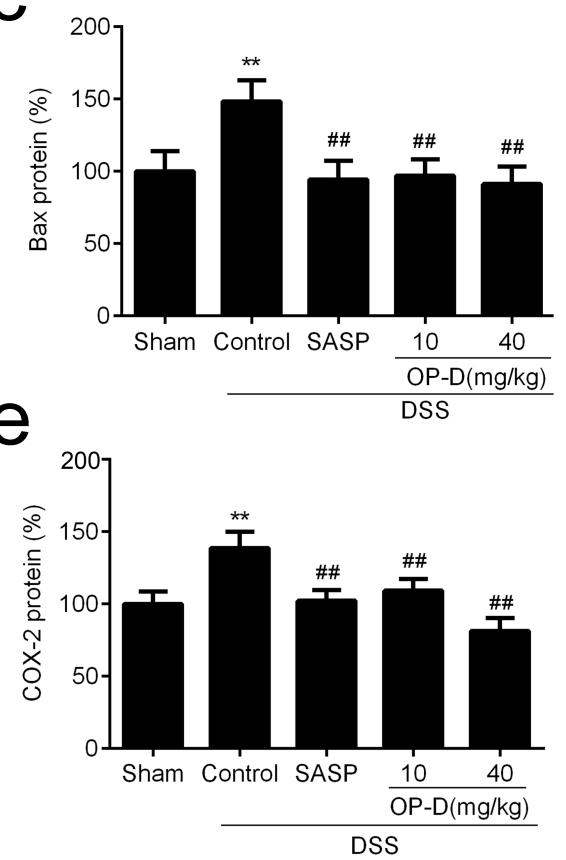

$\mathrm{h}$

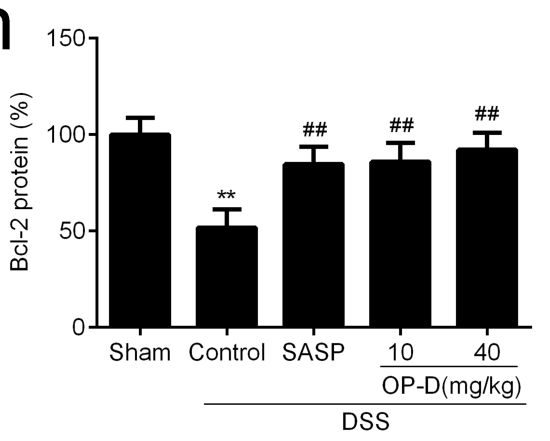

Figure 4

After seven days of OP-D (10 mg/ $\mathrm{kg}$ and $40 \mathrm{mg} / \mathrm{kg})$ treatment, the effects of OP-D on the expression of $\operatorname{MLCK}(a, b), \operatorname{Bax}(a, c)$, iNOS(a, d), COX-2 (a, e), cleaved caspase-3 $(a, f)$, occluding $(a, g)$ and Bcl-2 $(a, h)$ were examined by western blotting analyses. Data are expressed as the mean \pm SD. The data in the sham group is set to $100 \%$, and other data is set to the relative value of sham. ${ }^{*} P<0.05$, ${ }^{*} P<0.01$, compared to the sham group ( $n=6$ mice); $\# P<0.05, \# \# P<0.01$, compared to the control (DSS-colitis) group ( $n=6$ mice). 
a

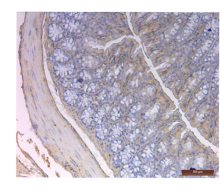

Sham

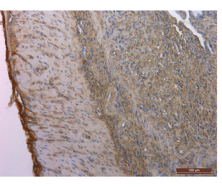

Control

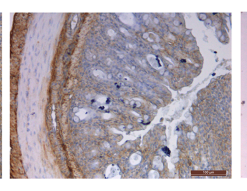

SASP

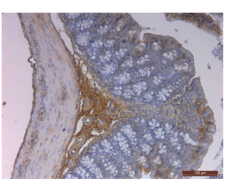

10

OP-D $(\mathrm{mg} / \mathrm{kg})$

DSS

C

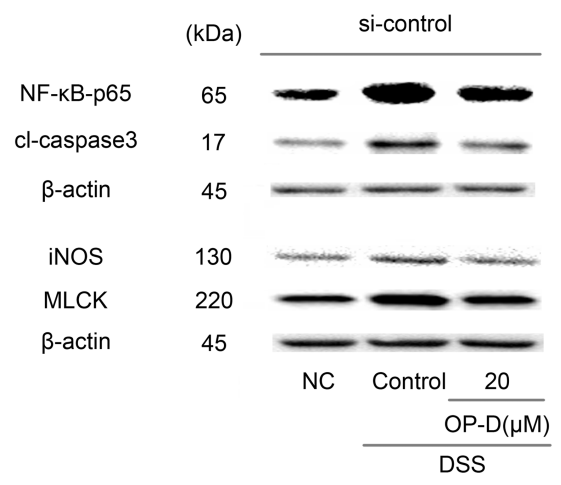

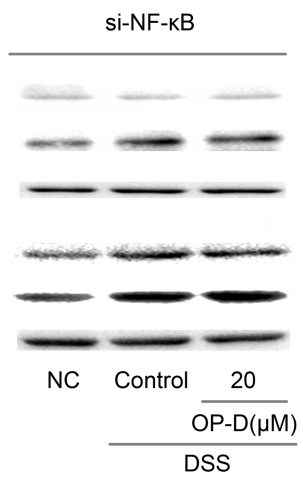

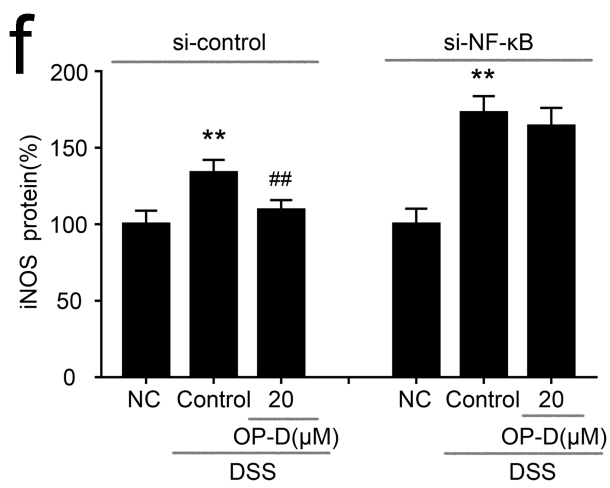

b

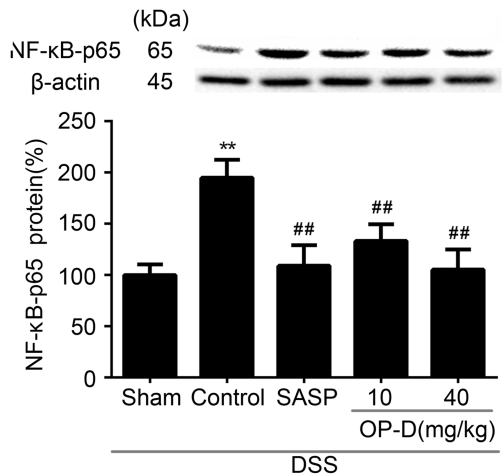

0

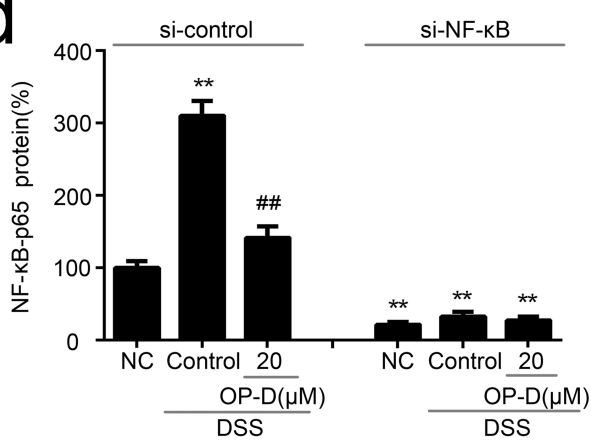

g

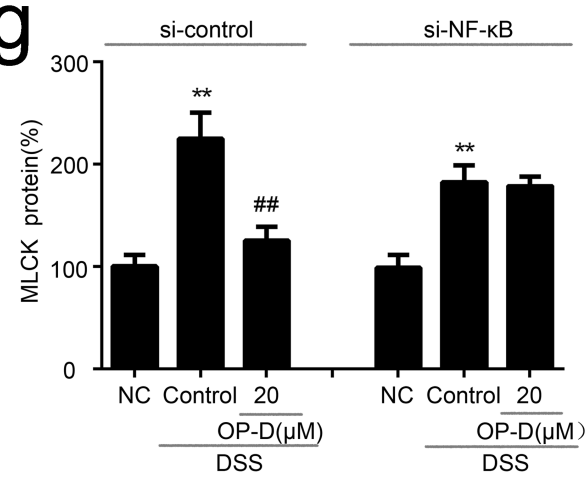

Figure 5

Potential mechanisms underlying the OP-D-induced treatment effects on colitis. Colonic segments were isolated after seven days of OP-D (10 mg/kg and $40 \mathrm{mg} / \mathrm{kg}$ ) treatment, (a) Immunohistochemical staining of NF-kB-p65, (b) Western blotting analyses of the inflammation marker NF-kB-p65. (c-g) Effects

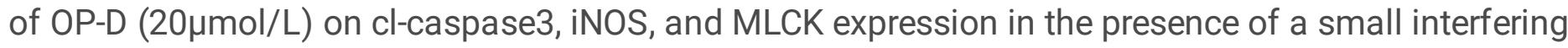
RNA targeting NF-KB-p65. (c) Representative western blotting images for the effects of OP-D on clcaspase3, iNOS, and MLCK expressions. Statistical analyses of OP-D on the expression of NF-KB-p65 (d), cl-caspase3 (e), iNOS (f), and MLCK (g). Data are expressed as the mean \pm SD. The data in the normal control (NC) group is set to $100 \%$, and other data is set to the relative value of $N C$. ${ }^{*} P<0.05,{ }^{\star} * P<0.01$, compared to the NC group ( $n=6$ samples); \#\#P<0.01, compared to the control (LPS) group ( $n=6$ mice). 


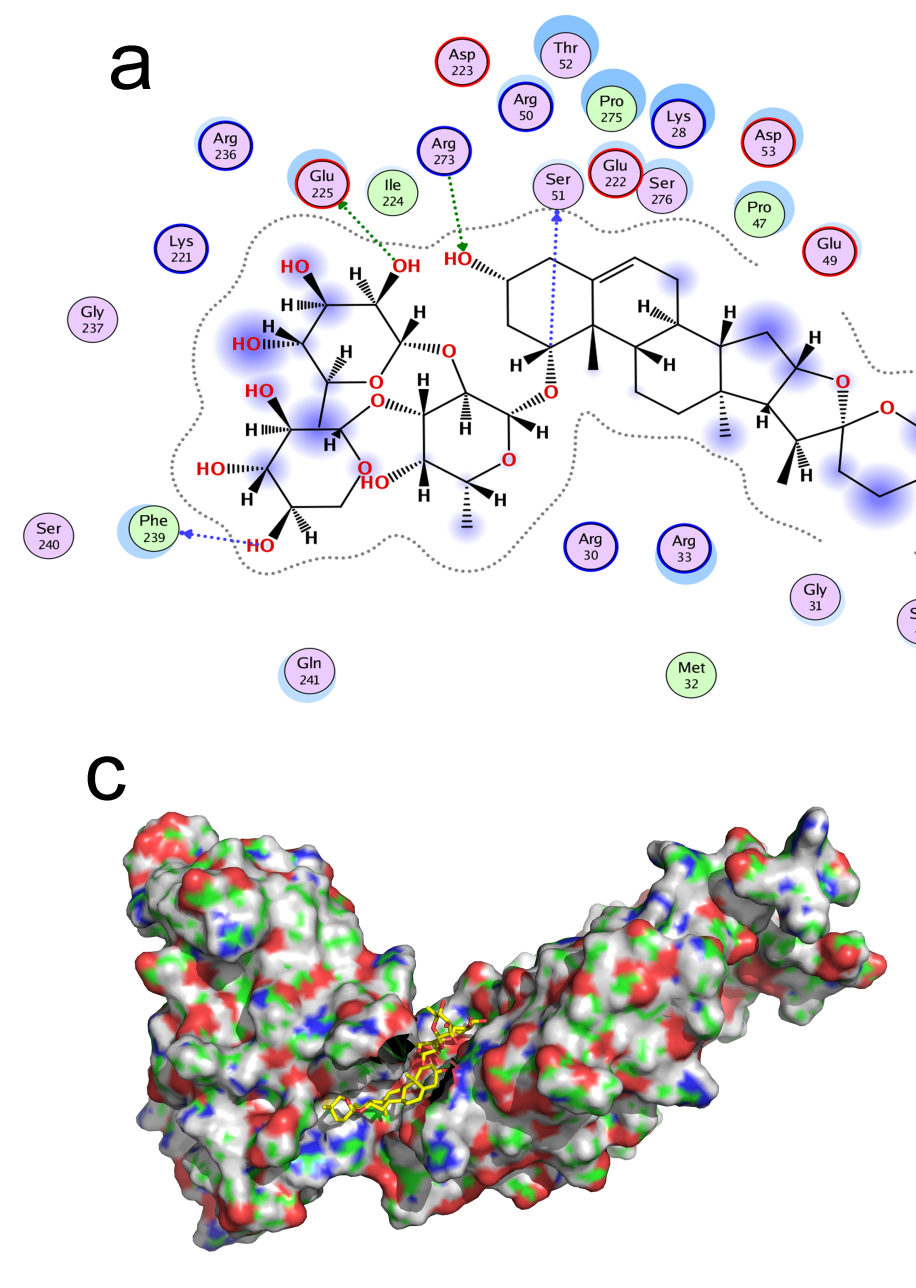

.
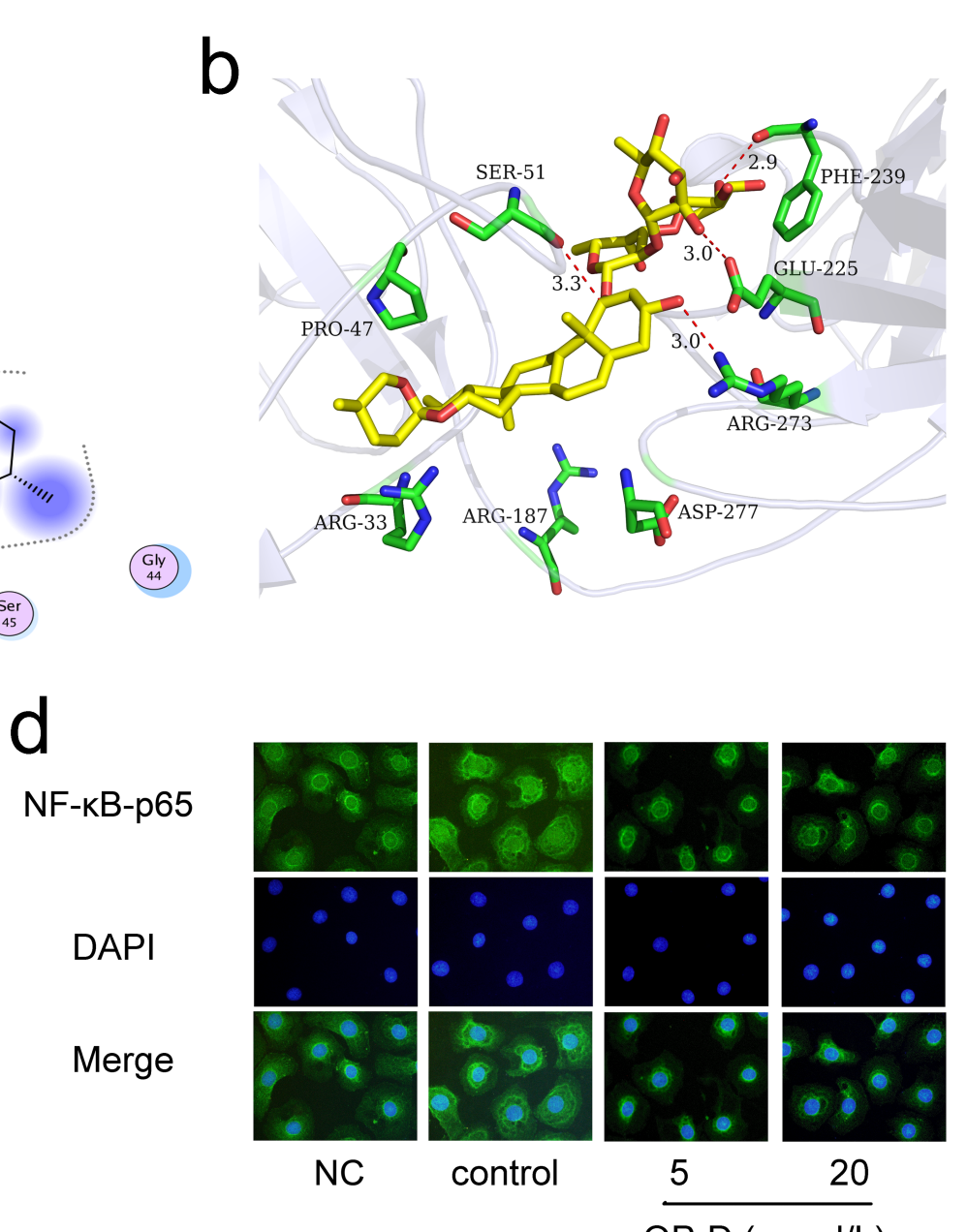

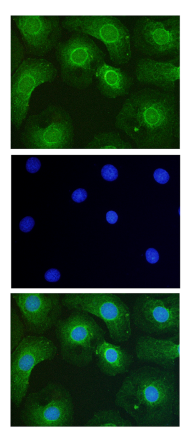

NC

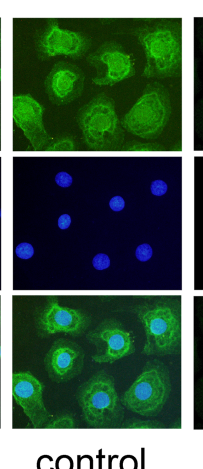

control

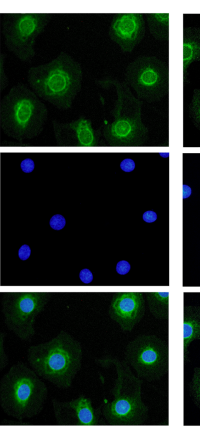

5

OP-D $(\mu \mathrm{mol} / \mathrm{L})$

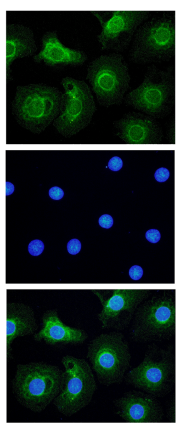

20

$\operatorname{LPS}(2 \mu \mathrm{g} / \mathrm{ml})$

\section{Figure 6}

Molecular docking analysis of the binding mode of OP-D with NF-KB-p65. (a) The 2D binding mode of OPD with NF-KB. (b) The 3D binding mode of OP-D with NF-KB-p65. The carbon atom of the saponin of OP-D was regarded as the hydrogen bond donor and formed a hydrogen bond with the oxygen atom of the backbone Ser51 in NF-KB-p65. The oxygen atom of the hydroxyl group of saponin of OP-D was regarded as the hydrogen bond acceptor and formed a hydrogen bond with the nitrogen atom of the guanidine group Arg273 in NF-kB-p65. The oxygen atom of the hydroxyl group of the rhamnose of OP-D was regarded as the hydrogen bond donor to form a hydrogen bond with the oxygen atom of the carboxyl group Glu225 in NF-KB-p65. The oxygen atom of the hydroxyl group of arabinose in OP-D was regarded as the hydrogen bond donor and formed a hydrogen bond with the oxygen atom of the backbone Phe239 in NF-KB-p65. (c) The surface binding mode of OP-D with NF-KB. OP-D is colored yellow, while the surrounding residues in the binding pockets are colored green. The backbone of the receptor is depicted as a light blue cartoon. (d) Effect of OP-D on translocation of NF-KB-p65 into the nucleus.

Immunofluorescence study indicated that OP-D could block the nuclear translocation of NF-KB-p65 in ICE6 cells stimulated by LPS『suggesting that OP-D has potential anti-inflammatory effect on IBD. 